\title{
Effects of deep brain stimulation amplitude on motor performance in Parkinson's disease
}

\author{
This article was published in the following Dove Press journal: \\ Journal of Parkinsonism \& Restless Legs Syndrome \\ 10 December 2012 \\ Number of times this article has been viewed
}

\author{
Alison Conovaloff' \\ Narayanan Krishnamurthi' \\ Padma Mahant ${ }^{2}$ \\ Johan Samanta ${ }^{2}$ \\ James Abbas' \\ 'Center for Adaptive Neural Systems, \\ School of Biological and Health \\ Systems Engineering, Ira A Fulton \\ School of Engineering, Arizona \\ State University, Tempe, AZ, USA, \\ ${ }^{2}$ Movement Disorders Clinic, Banner \\ Good Samaritan Medical Center, \\ Phoenix, AZ, USA
}

Background: The efficacy of deep brain stimulation (DBS) in Parkinson's disease has been convincingly demonstrated in studies comparing motor performance with and without stimulation, but characterization of the stimulation dose-response curves has been limited.

Methods: In a series of case studies, eight subjects with Parkinson's disease and bilateral DBS systems were evaluated at their clinically determined stimulation (CDS) and at three reduced amplitudes, ie, approximately $70 \%, 30 \%$, and $0 \%$ of the CDS (MOD, LOW, and OFF, respectively). Performance was assessed using the motor section of the Unified Parkinson's Disease Rating Scale (UPDRS-III), which includes subscores for tremor, bradykinesia, gait, posture, and tapping. Data at the reduced settings were analyzed to determine if individual subjects demonstrated a threshold-like response, which was defined as a dose-response curve in which one decrement in stimulation accounted for $\geq 70 \%$ of the maximum change observed. Day-to-day variability was assessed using the CDS data from the three different days.

Results: In the dose-response curves, two subjects exhibited a threshold-like response, four exhibited a graded change, and two did not exhibit substantial changes. For some subjects, variability in CDS performance across the three days exceeded the change observed when reducing amplitude to the MOD setting. Comparisons across this set of eight subjects demonstrated that the mean UPDRS-III and all but one subscore significantly increased (performance degraded) when amplitude was reduced from CDS to the LOW and OFF conditions, but there were no significant changes when amplitude was reduced from CDS to the MOD condition.

Conclusion: Individual differences in the DBS dose-response curves may provide opportunities to optimize clinical performance. Day-to-day variability in motor performance cautions against the use of a single UPDRS measurement in clinical selection of DBS settings.

Keywords: deep brain stimulation, Parkinson's disease, Unified Parkinson's disease rating scale, motor performance

\section{Introduction}

For many people with Parkinson's disease, deep brain stimulation (DBS) of the subthalamic nucleus can alleviate many symptoms of the disease, reduce levels of prescribed medication, and reduce medication-related side effects. ${ }^{1-4}$ Several studies have compared motor performance at patients' clinically determined stimulation (CDS) settings, ie, the settings currently considered by the patient's clinician to be optimal for the patient, with performance while the DBS system is turned off com- 
pletely. ${ }^{5-9}$ Although such studies demonstrate the benefits of using DBS, they do not provide much guidance to the clinician trying to select stimulation parameters that balance the tradeoffs between clinical effects and battery life. A more detailed understanding might help clinicians select more suitable stimulation parameters, but only a few studies have begun to investigate motor performance at intermediate stimulation settings. For example, one study investigated the effects of different combinations of contact location (relative to the subthalamic nucleus) and amplitude in patients with Parkinson's disease. Given that the main focus was speech intelligibility, movement was only examined as a composite outcome. ${ }^{10}$ Another demonstrated that frequency and stimulation amplitude were the most important factors in alleviating tremor amplitude in patients with essential tremor, while varying pulse width had little effect. ${ }^{11}$ In both studies, patients were evaluated at the same amplitude values regardless of the settings that had been prescribed specifically for each patient.

The effects of varying pulse width, frequency, and stimulation amplitude on tremor, rigidity, and bradykinesia have been investigated in one study, ${ }^{12}$ but it did not evaluate effects on posture or gait. This report also concluded that varying pulse width did not have a clear effect on symptoms of Parkinson's disease, while stimulation amplitude was the most important factor in alleviating the parkinsonian triad.

In the series of case studies reported here, we evaluated five components of motor performance, ie, tremor, bradykinesia, gait, posture, and tapping, in a population with Parkinson's disease. Based upon the reports cited above, ${ }^{11,12}$ we focused our assessment on the effect of changing stimulation amplitude only. Motor performance was assessed using the motor section of the Unified Parkinson's Disease Rating Scale (UPDRS-III). A UPDRS subscore was defined and assessed for each component. Given that the CDS amplitude setting can vary widely across individuals, we varied amplitude as a percentage of the CDS value to facilitate comparison across subjects. The CDS amplitude was defined as the ceiling, because stimulation amplitude is often set just below the threshold for adverse effects. ${ }^{12}$

Symptoms are known to vary daily in people with Parkinson's disease, with studies indicating that consecutive UPDRS measurements may vary by as much as $15 \%$, yet many DBS studies have compared capabilities as assessed on different days. ${ }^{713}$ Therefore, we also examined motor performance at the same stimulation condition over 3 different days to provide a daily baseline measurement.

\section{Materials and methods}

\section{Subjects}

Subjects who met the following criteria were recruited from the Movement Disorders Clinic at Banner Good Samaritan Medical Center and Movement Disorder Specialists (Phoenix, AZ): age 18-80 years; diagnosis of idiopathic Parkinson's disease with bilateral symptoms; a Hoehn and Yahr stage $\leq 4$ during the "medication-on/stimulation-on" condition; bilateral DBS system implanted in the subthalamic nucleus for more than 3 months; willingness to sign the informed consent document; and ability to understand and follow directions.

Subjects were excluded if they presented with any of the following: significant hepatic, renal, cardiovascular, endocrine, respiratory, or unstable neurological disease aside from Parkinson's disease; psychotic illness or chronic psychiatric disorder; history of drug dependence or intellectual impairment; history of cerebral insult (causing delayed secondary Parkinson's disease); a Hoehn and Yahr stage of 5 during the "medication-on/stimulation-on" condition; Parkinson's plus syndrome; score $\geq 3$ for UPDRS-II items 13-15 (falling unrelated to freezing, freezing when walking, and walking) during the "medication-on/stimulation-on"condition; or score $\geq 4$ during the "medication-on/stimulation-on" condition for UPDRS III items.

Eight subjects (six males, two females) met the criteria and were enrolled in the study. The mean age at enrollment was $59.5 \pm 8.9$ years. Mean time since diagnosis of Parkinson's disease was $12.6 \pm 4.0$ years, and mean time since the first DBS surgery was $46.8 \pm 23.7$ months. The median Hoehn and Yahr stage at enrollment was 2.0, with a range of 2.0-3.0 (Table 1). Seven of the subjects had previously been implanted with two Soletra ${ }^{\circledR}$ model 7426 neurostimulators (Medtronic Inc, Minneapolis, MN), while one subject (subject 2) had previously been implanted with one dual-program Kinetra ${ }^{\circledR}$ model 7428 neurostimulator (Medtronic Inc). The surgical placement technique used for all subjects included the use of microelectrode recordings. All subjects had been implanted with model 3387 macroelectrodes (Medtronic Inc) except subjects 4 and 8 , who had been implanted with model 3389 macroelectrodes (Medtronic Inc).

\section{Experimental protocol}

For each subject, data were collected during three separate sessions, with a $1-4$-week period between sessions (actual mean time between sessions $16.6 \pm 5.0$ days). All sessions were conducted at the Clinical Neurobiology and Bioengineering Research Laboratory at Banner Good 


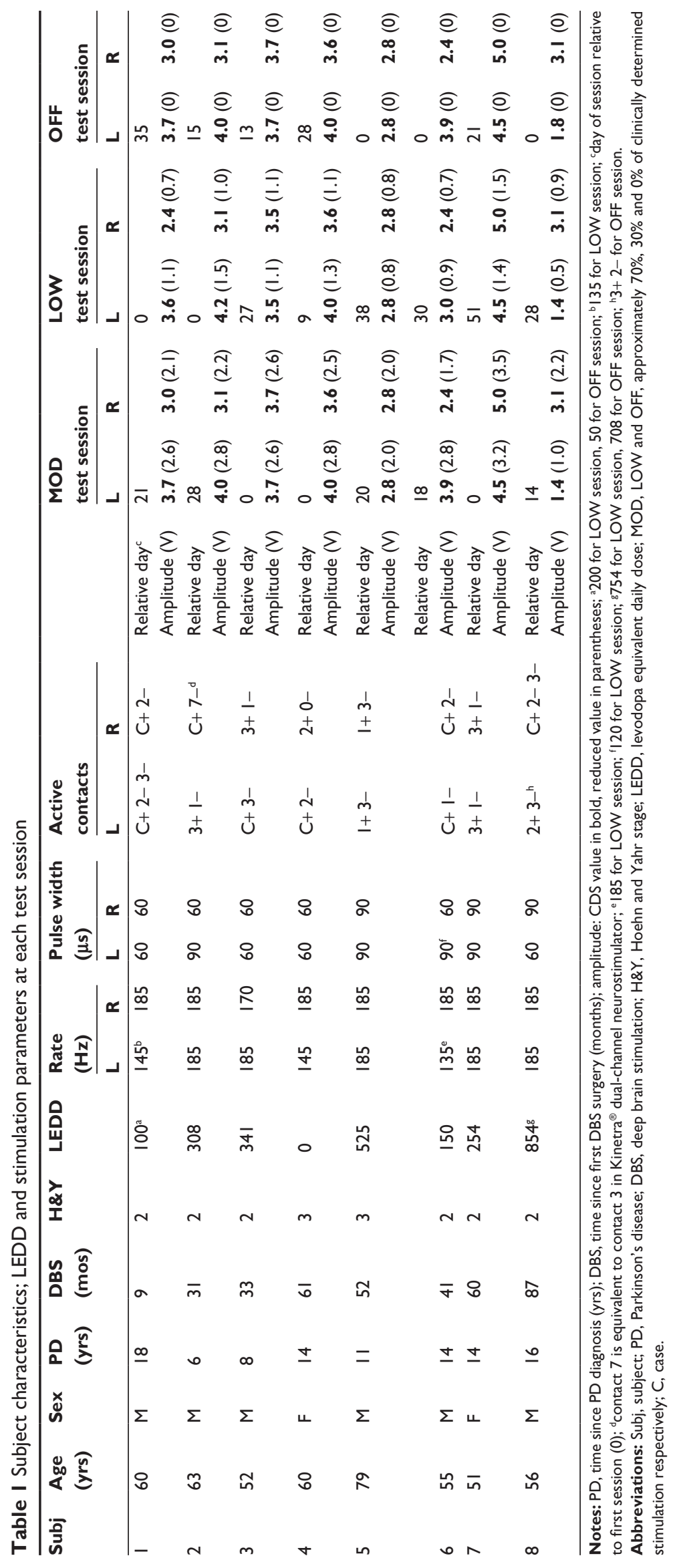


Samaritan Medical Center in Phoenix, AZ. All experimental procedures were approved by the center's institutional review board and conducted in accordance with the center's guidelines and the Declaration of Helsinki. Informed consent was obtained from all subjects before participating in this research.

The experimental sessions were conducted during subjects" "medication-on" state in order to provide information about the dose-response characteristics of DBS as a supplement to prescribed medication. Although several studies have defined the "medication-on" state as a suprathreshold dosage of levodopa, here the "medication-on" state represented the subjects' daily living conditions to simulate a clinically relevant situation and allow the results to be readily transferred to the clinic. ${ }^{14}$ To ensure subjects were at the same point in their medication cycle, all three sessions occurred at the same time of day for each subject. The dosage of medication is reported as the levodopa equivalent daily dose in Table $1 .{ }^{15}$ Of note, one subject (subject 4) was not taking any antiparkinsonian medications throughout the course of the test sessions.

At each session, subjects were first evaluated at their currently programmed CDS settings and next with reduced amplitude stimulation settings. This provided a consistent assessment of change in the same direction (ie, always from CDS to the altered condition) and a consistent assessment of the CDS settings (ie, it was always assessed before any changes to stimulation occurred). Reduced settings were always chosen relative to each subject's CDS amplitude on that day, therefore allowing performance at reduced settings to be compared with performance at the settings currently determined to be optimal by the patient's clinician. Subjects were given a 20-minute rest period after their DBS settings were reduced to allow any changes induced by the reduction to take full effect, which is equivalent to or longer than the calibration time in other studies that have evaluated motor performance at multiple stimulation settings. ${ }^{10-12,16}$ This rest period also limited the effects of subject fatigue and/or changes in drug efficacy that might have occurred with longer rest periods. The reduced amplitude setting on a given day was one of three conditions: MOD (approximately 70\% of CDS), LOW (approximately 30\% of CDS), and OFF (DBS stimulation completely switched off). Given the limited resolution on the implanted pulse generator, stimulation conditions were selected to be as close as possible to the target percent condition. Across subjects, the order in which the reduced amplitude settings were tested was randomized.
The CDS and reduced amplitude values, rate, pulse width, and active contacts used for each subject during each test session are given in Table 1. The mean CDS, MOD, and LOW amplitudes were $3.4 \pm 0.8 \mathrm{~V}, 2.4 \pm 0.6 \mathrm{~V}$, and $1.0 \pm 0.3$ $\mathrm{V}$, respectively. The mean rate was $177 \pm 17 \mathrm{~Hz}$. The mean pulse width was $74 \pm 16 \mu \mathrm{sec}$. Note that the study did not place any restrictions on changes in clinical prescription of medication or stimulation settings; those changes are noted in Table 1 and in the following sections of the text. In order to limit the effect of such intersession changes, the study primarily uses intrasession differences (between CDS and the altered condition) to characterize the response to changes in stimulation amplitude.

A trained DBS system operator queried the implanted pulse generator using a Model 8840 N'Vision ${ }^{\mathrm{TM}}$ clinician programmer (Medtronic Inc), recorded the current stimulation settings, and changed the stimulation settings. A researcher trained to administer the UPDRS evaluated items 18-31 (the motor examination section) during all experimental sessions.

\section{Outcome measures}

Outcome measures included the total UPDRS III motor score (maximum 108) along with several motor subscores. Note that on this scale, a higher score indicates worse performance. For individual subjects, a change of five points in the total UPDRS III motor score ( $4.6 \%$ of the maximum score) was considered to be a minimal clinically important change, as defined by Schrag et al (for patients in Hoehn and Yahr stages $1-3) .{ }^{17}$

All of the subscores except for tapping were based on definitions used in previous studies: tremor $=$ items 20-21 assessed only at the hands (maximum 16); ${ }^{18,19}$ bradykinesia $=$ items $23-26,31($ maximum 36$),{ }^{20}$ gait $=$ item 29 (maximum 4$) ;{ }^{21}$ and posture $=$ items 28,30 (maximum 8$) .{ }^{22}$ The tapping subscore was defined as item 23 (maximum 8). The differences in mean scores obtained at the CDS and reduced amplitudes were calculated for each outcome measure and normalized by the maximum possible score for that particular measure to facilitate comparison across different measures. For each of the subscores, we have identified which measures exhibited a change $>25 \%$ of the maximum possible score.

\section{Dose-response characteristics}

To characterize the nature of the response to stimulation, dose-response curves were produced for the overall UPDRS III and for each of the subscores. In these plots, 
dosage is reported as stimulation condition, which was normalized by the CDS amplitude for each subject. The response is reported as the change in the UPDRS score (or subscore) recorded at each altered stimulation amplitude with respect to the score (or subscore) recorded at the CDS condition of stimulation on that day.

The linearity of the dose-response curves for each subject was assessed by calculating the slopes of each of the segments. If the response to stimulation was graded and linear, each decrement in stimulation would account for $30 \%-40 \%$ of observed maximum change in outcome measure (30\% for MOD, $40 \%$ for LOW, and 30\% for OFF). If the response exhibited a purely threshold (on/off) effect, one of the decrements in stimulation would account for $100 \%$ of the change in outcomes. In this analysis, a subject was characterized as having a "threshold" dose-response characteristic if one decrement in stimulation accounted for $\geq 70 \%$ of the maximum performance change observed, which is the amount of change expected from two decrements. Otherwise, the subject was characterized as having a "graded" dose-response characteristic.

\section{Statistical analysis}

Statistics were performed using IBM SPSS Statistics software version 20 (SPSS Inc, Chicago, IL). For each outcome measure, the two-tailed Wilcoxon signed rank test was used to compare the values measured at each session's CDS amplitude with the values measured at that same session's reduced amplitude. Performance at each session's CDS amplitude was treated as a baseline measure for performance at the associated reduced amplitude to account for minor changes in medication or CDS settings over the course of all three test sessions. Unless otherwise noted, summary data is reported as the mean \pm one standard deviation, and a $P$ value $<0.05$ was considered to be statistically significant.

\section{Results \\ Dose-response characteristics}

Although the mean dose-response curves for the subscores (Figure 1) were nearly linear, there was high variability across subjects. Applying the linearity criterion described above to the total UPDRS III motor score, four subjects exhibited a graded dose-response characteristic (3, 4, 6, and 7) and two exhibited a threshold response ( 1 and 2 ). The two subjects (5 and 8 ) who showed a very small overall change in the UPDRS III score were not included in this characterization of the dose-response curve.

\section{Comparison across stimulation conditions}

When amplitude was reduced from the CDS to the MOD condition, only four subjects experienced a clinically important decrease in performance, and two of these decreases were only borderline, defined as 5-7 points (Figure 2, Table 2). However, all eight subjects experienced a clinically important change ( $\geq 5$ points) when amplitude was reduced from the CDS to the LOW condition (three designated as borderline changes). When amplitude was reduced to the OFF condition, six subjects showed clear deterioration in performance with increases in the total UPDRS III motor score ranging from 13-59 points. Interestingly, two of the subjects ( 5 and 8 ) who only experienced borderline changes in the LOW condition did not experience a clinically important change in the OFF condition.

For the subscores, none of the subjects experienced change $>25 \%$ of the maximum score at the MOD condition, but half of the subjects experienced a change $>25 \%$ of the maximum score for at least one subscore at the LOW or OFF conditions. The other four subjects $(3,4,5$, and 8$)$ did not experience a change $>25 \%$ of the maximum score in any condition.

When the subjects were examined as a group, none of the mean outcomes degraded significantly when amplitude was decreased from the CDS to the MOD condition (Figure 3). However, all mean outcomes except one significantly degraded when amplitude was decreased from the CDS to the LOW and OFF conditions (gait and posture, respectively).

\section{Repeatability at CDS condition}

Each subject had three scores obtained at the CDS condition, one from each data collection session (Table 2). For the total UPDRS III motor score, the difference in CDS measurements was calculated to determine if there were any clinically important changes between days. Subjects 3, 5, 6, and 7 experienced a clinically important change between at least two sessions. For the subscores, the range of these three measurements was calculated to characterize the variability in each subject's results (Figure 1). Compared with the maximum possible score for each subscore, subjects 1 , 2 , and 8 experienced low variability for all of the subscores. Subjects 3, 4, 6, and 7 experienced low variability on all but one subscore, and subject 5 experienced high variability for two subscores.

The range of CDS values was also compared with the change in score from the CDS condition to each reduced 

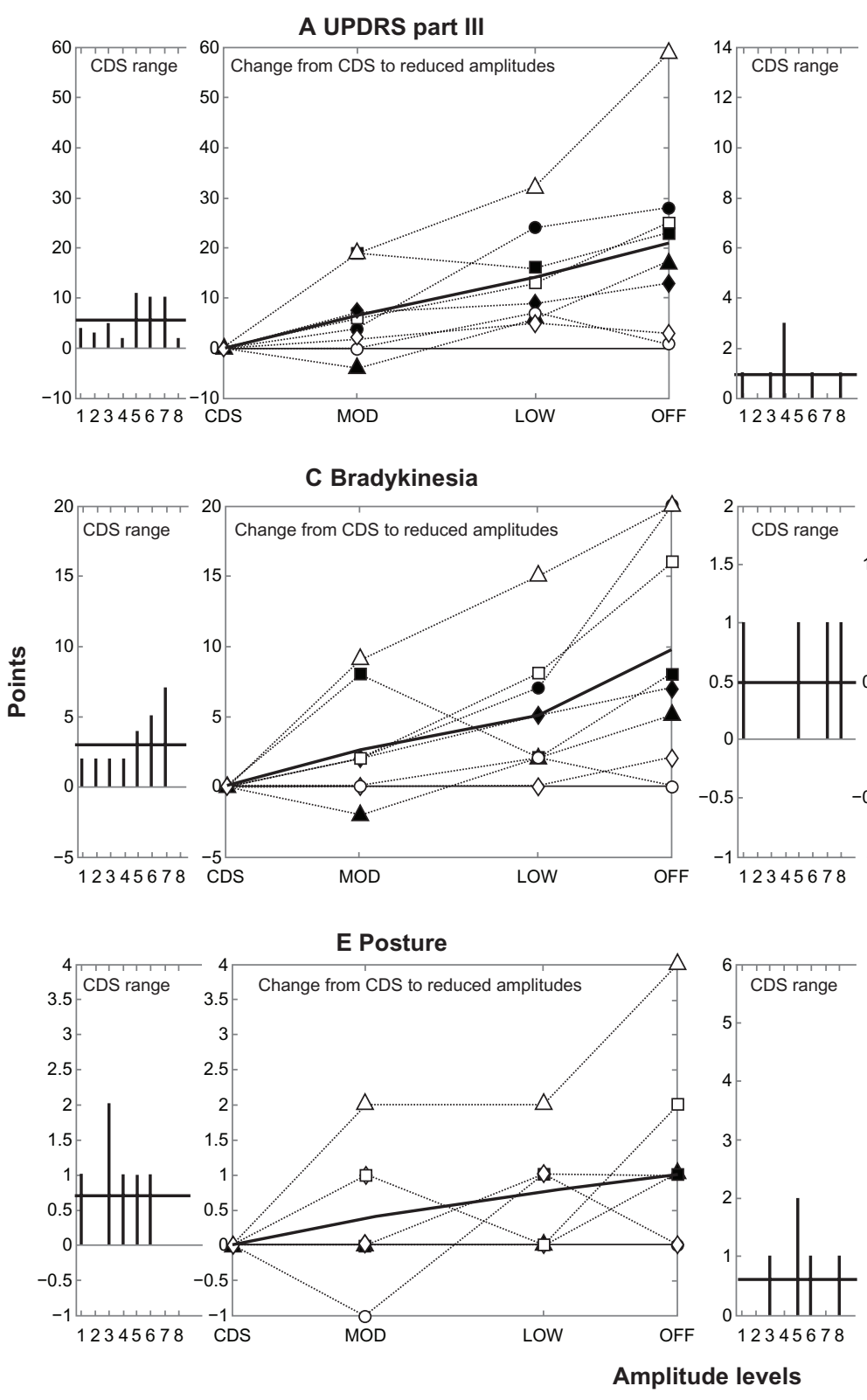

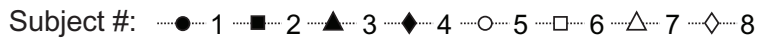
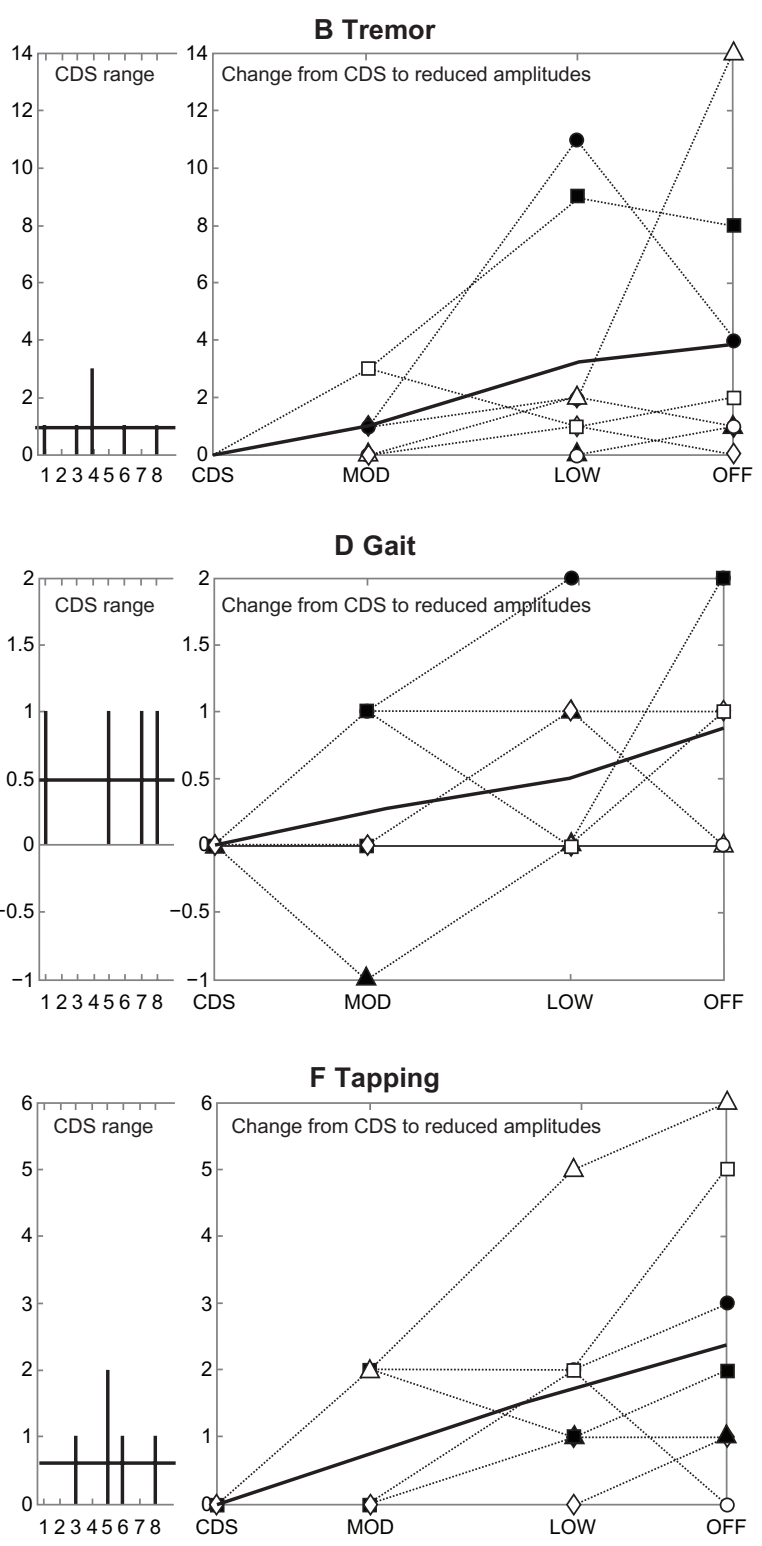

Figure I Day-to-day CDS variability compared to change in outcome measure from CDS to each reduced amplitude.

Notes: Left side of each lettered subplot represents each subject's range of CDS values over the three sessions for (A) UPDRS Part III score, (B) tremor subscore, (C) bradykinesia subscore, (D) gait subscore, (E) posture subscore, and (F) tapping subscore.

The horizontal line represents the mean, while the vertical bars represent each individual subject. Right side of each lettered subplot represents change in outcome measure from CDS to each reduced amplitude for (A-F). The black line represents the mean, while the gray lines represent each individual subject.

Abbreviations: CDS, clinically determined stimulation; UPDRS, Unified Parkinson's Disease Rating Scale; MOD, LOW and OFF, approximately 70\%, 30\% and 0\% of CDS respectively.

amplitude condition. The mean change in score from the CDS to the MOD condition was comparable with the mean CDS range for the total UPDRS III score as well as for the tremor, bradykinesia, and tapping subscores, but the mean changes in score from the CDS to the LOW and OFF conditions were greater than the mean CDS range. However, for the gait and posture subscores, the mean change in score from the CDS to the MOD condition was half the size of the mean CDS range, and the mean change in score from the CDS to the LOW condition was equal to the mean CDS range. For these two subscores, only the mean change in score from the CDS to the OFF condition was greater than the mean CDS range.

When examined for each subject, the change in score from the CDS to the MOD condition was greater than the CDS range for subjects 2 and 7 for the total UPDRS III score as well as the majority of the subscores. For the rest of the subjects, the change in score from the CDS to the 

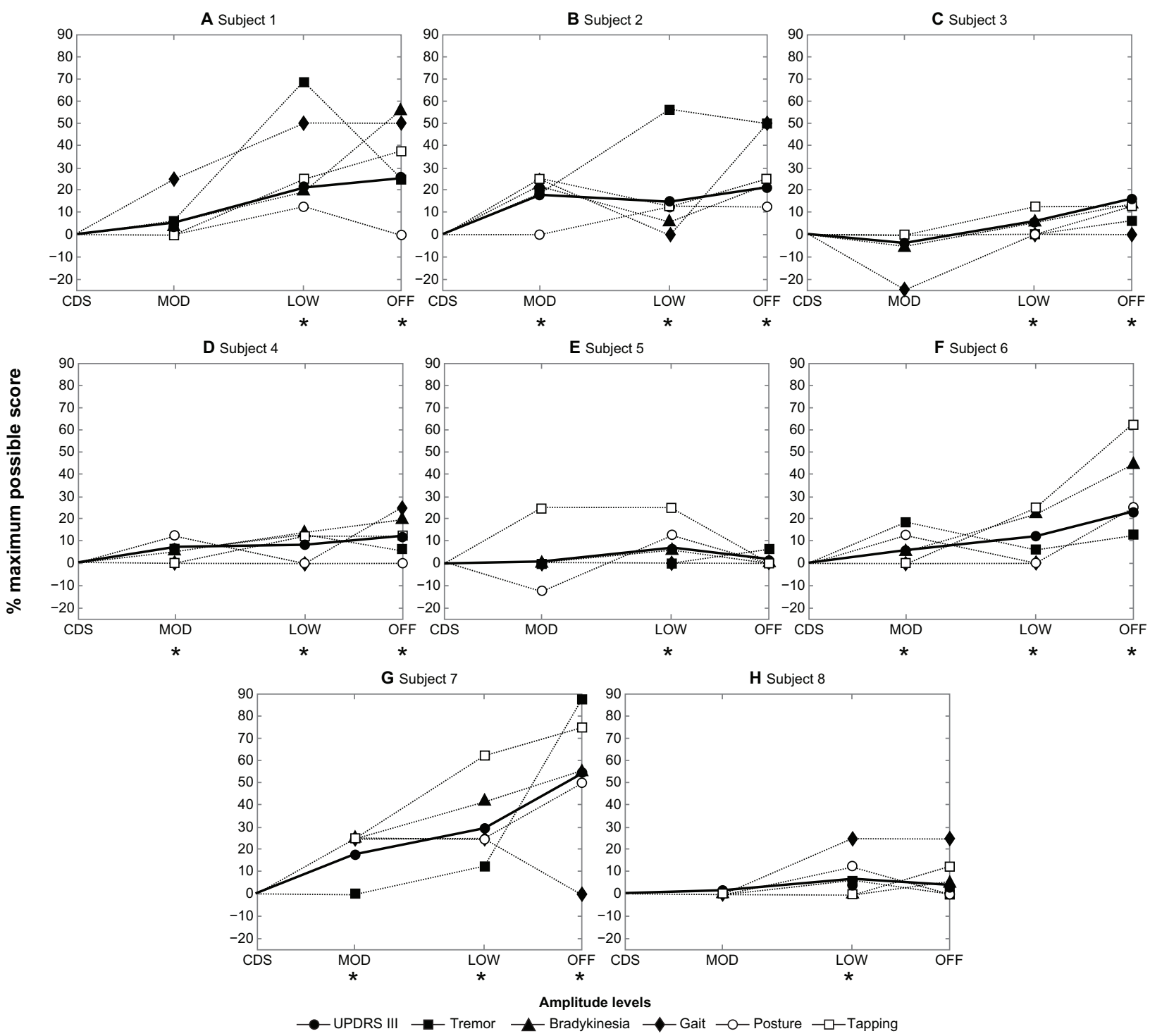

Figure 2 Change in outcome measure from CDS to each reduced amplitude as a percentage of the maximum possible score for that particular measure for each subject. Notes: The black line represents the UPDRS Part III score, while the gray lines represent each subscore. *Indicates clinically important changes in the UPDRS Part III scores observed at CDS and reduced amplitude during the same test session.

Abbreviations: CDS, clinically determined stimulation; UPDRS, Unified Parkinson's Disease Rating Scale; MOD, LOW and OFF, approximately $70 \%$, 30\% and 0\% of CDS respectively.

MOD condition was comparable with or less than the CDS ranges for the total UPDRS III score and the majority of the subscores. In contrast, the change in score from the CDS to the LOW condition exceeded the CDS range for the total UPDRS III score for all subjects except subject 5 and for at least half of the subscores for subjects 1,2 , 4,6 , and 7 .

\section{Discussion}

\section{Individual subject measures}

Subjects 1 and 2 were both characterized as having a threshold in the dose-response curve. In addition, neither exhibited clinically significant day-to-day variability in the
CDS measures. However, subject 2 experienced a greater change in score when amplitude was reduced to the MOD condition compared with the range of scores experienced during different CDS sessions, while subject 1 did not. This difference may be explained by the location of the thresholds for the two subjects. The threshold for subject 1 was located between the MOD and LOW conditions, with little difference observed between CDS and MOD. However, the threshold for subject 2 was located between CDS and MOD and a reduction in stimulation amplitude of $30 \%$ accounted for more than $80 \%$ of the total observed change in score.

Subjects 3, 6, and 7 were all characterized as having a graded dose response curve and all exhibited some clinically 


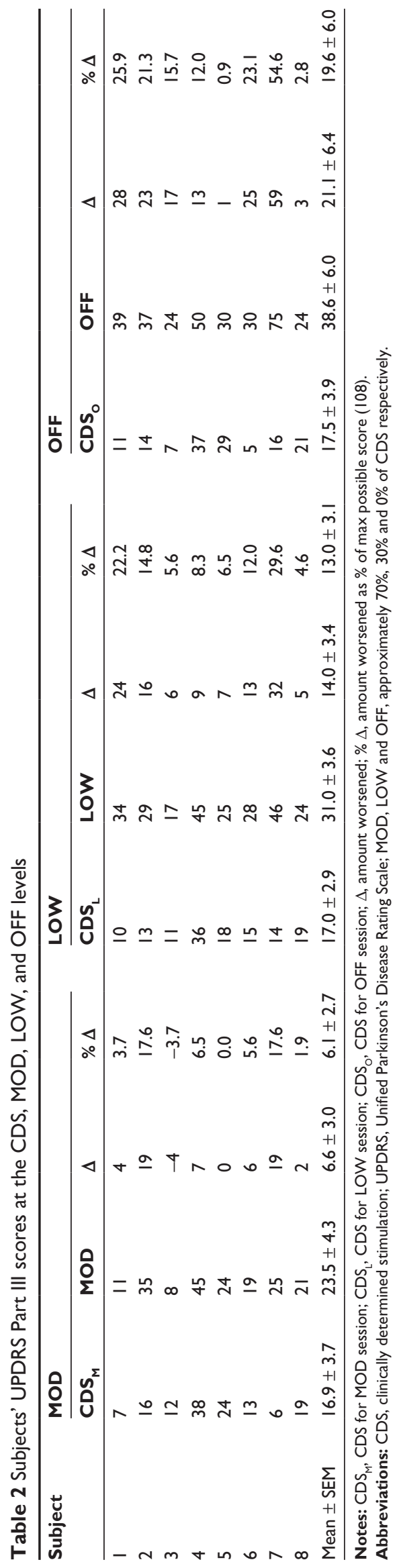

significant variability in the CDS outcomes. For these subjects, the graded response characteristic may be responsible for observed day-to-day variability as well as the sensitivity to changes in stimulation. The characteristics of these subjects demonstrate that at least some patients may benefit from fine-tuning of the stimulation level and possibly may benefit from a system that allows for daily adjustments to stimulation level either in an automated or patient-selected manner. However, subject 4 was also characterized as having a linear dose-response curve but did not exhibit clinically significant variability in CDS outcomes. This observation may be due to the fact that the total effect of stimulation was low in this subject and this was the only subject in the study who was not taking antiparkinsonian medications. Subjects 5 and 8 demonstrated less sensitivity to the degree of stimulation reduction compared with the other subjects, with the largest observed changes in the total UPDRS III score at less than 10 .

\section{DBS dose-response characteristics}

Several studies have demonstrated the value of DBS by assessing motor function with and without stimulation, but very few have investigated more subtle changes in stimulation values. By assessing motor function at four different amplitudes, we have been able to characterize the dose-response curves for individual subjects. With only four data points, this is a very coarse characterization of the dose-response curve, but does enable classification of different response characteristics that may have implications for clinical adjustments to stimulation settings.

A linear dose-response curve would suggest that the effect of DBS could be precisely regulated by adjusting stimulation amplitude. Although the group means for the total UPDRS III score and each of the subscores all exhibited linear dose-response curves, the data demonstrated a high degree of variability across the set of subjects. These data reflect the variability observed clinically in the effects of Parkinson's disease on motor performance and the variability in the reported on/off effects of DBS. Although a wide range of response characteristics was demonstrated across symptoms, six of the eight subjects could be grouped into one of two categories, comprising those who exhibited a threshold, ie, an all-or-none type of response to stimulation and those who exhibited a graded response that was approximately linear.

The two classes of dose-response characteristics that were observed may be due to differences in the nature and/ or stage of Parkinson's disease or may be due to differences in electrode location. If this classification scheme were to be 

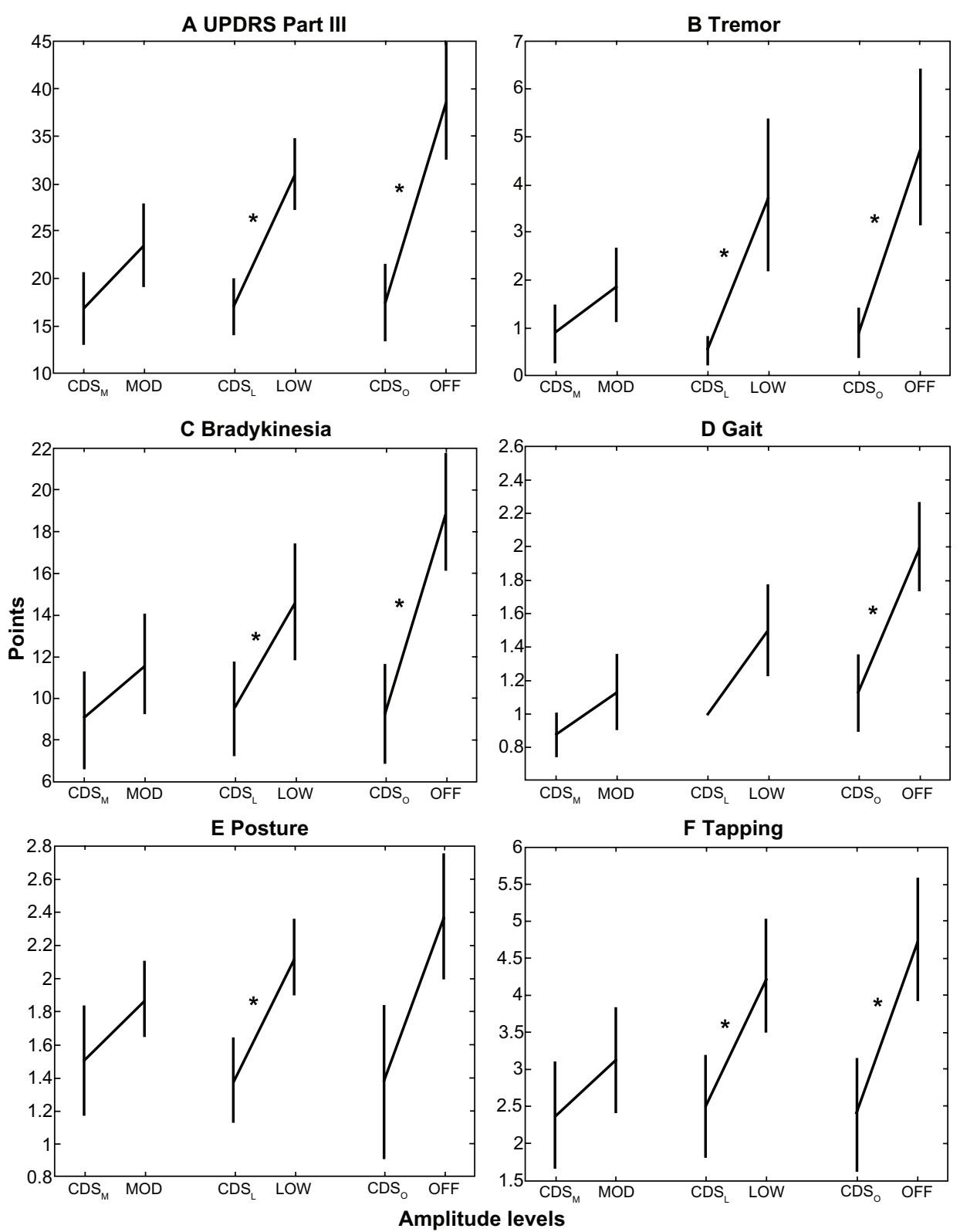

Figure 3 Changes in mean ( \pm standard error of the mean) UPDRS score/subscores from the CDS condition for each amplitude level. *Indicates changes in mean across subjects of each outcome that were significant. (A) UPDRS Part III score, (B) tremor subscore, (C) bradykinesia subscore, (D) gait subscore, (E) posture subscore, and (F) tapping subscore.

Note: $C_{M}$, $C D S_{L}$, and $C D S_{O}: C D S$ amplitudes for the sessions in which amplitude was reduced to the MOD, LOW, and OFF levels, respectively.

Abbreviations: CDS, clinically determined stimulation; UPDRS, Unified Parkinson's Disease Rating Scale; MOD, LOW and OFF, approximately 70\%, 30\% and 0\% of CDS respectively.

confirmed in a larger study, the results could lead to the development of alternative parameter selection procedures for use in the clinic: those exhibiting graded responses could have stimulation levels iteratively adjusted to improve performance while those who exhibit threshold-like responses could have stimulation adjusted to just above their threshold in order to reduce battery power consumption. Perhaps more importantly, a larger study could also characterize electrode location to determine the degree to which dose-response classification is determined by surgical placement.

\section{Comparison across stimulation conditions}

Reducing the stimulation to the MOD condition caused a clinically important decrease in performance for half of the subjects, but further reductions (to the LOW and/or OFF setting) caused clinically important changes for all subjects. The lack of observed change at the MOD condition may truly reflect a lack of clinical effect or it may indicate a failure to detect the effect due to insufficient sensitivity in measurement procedures. Although the UPDRS III has 
been found to be reliable and valid, it has been criticized for placing too much emphasis on severe manifestations of symptoms and not enough on milder presentations, such as might be seen if stimulation amplitude was reduced by only $30 \% .^{23}$ In addition, the scale measures symptoms that are not experienced by every patient, which decreases its overall sensitivity. ${ }^{24}$ However, if there was indeed no significant change in performance at the MOD condition for some of the subjects, then stimulation amplitude could be reduced by at least $30 \%$ for these subjects and performance would not degrade.

Although many subjects experienced similar increases in their total UPDRS III motor scores and several even had similar raw scores at each amplitude condition, their performance as measured by the subscores was very different. This may be explained by the differences in the subjects' primary symptoms. Previous reports ${ }^{25}$ observed that although two patients may have the same UPDRS score, their quality of life may actually be very different depending on their prominent symptom(s), which suggests that adjusting stimulation parameters based on the total UPDRS III motor score alone may not always provide the most benefit for a patient's functionality.

In current clinical practice, stimulation amplitude is often chosen primarily based on the presentation of tremor, because tremor responds almost instantaneously to changes in stimulation. Unfortunately, tremor has been shown not to be correlated with other components of motor performance, to have little relation to functional disability, and to have little impact on patient quality of life. ${ }^{25-27}$ It is possible that stimulation amplitude should be set based on the response of other components of motor performance. Bradykinesia, postural stability, gait, and rigidity ratings have been suggested to be the most dominant factors contributing to disability in patients with Parkinson's disease. ${ }^{27}$

\section{Day-to-day variability}

Across the three sessions, four of the subjects $(1,2,4,8)$ exhibited consistent UPDRS III motor scores at the CDS amplitude, and four subjects $(3,5,6,7)$ exhibited a range of CDS scores that exceeded the criterion for minimal clinically important change. For comparison, the change in stimulation to the MOD condition produced a similar result: four subjects exhibited no clinically important change and four exhibited clinically important changes. The fact that half of the subjects exhibited clinically significant differences across days with the same stimulation settings indicates that day-to-day variability in motor performance is substantial and strongly cautions against the use of a single UPDRS reading in setting DBS parameters.

This study did not restrict changes in clinical prescription of stimulation or medication over the course of the subject's participation in the study, but it is important to note that these changes did not coincide with any of the observed clinically significant day-to-day variability in CDS scores. For subjects 3 and 6, although there were no changes in the CDS levels between sessions 1 and 2, there were clinically significant changes in the total UPDRS III scores observed at the CDS settings (from 12 to 7 for subject 3; from 5 to 13 for subject 6). Between sessions 2 and 3, the CDS levels had been changed for both subjects (values reported in Table 1), but there was only a small change in UPDRS score (from 7 to 11 for subject 3; from 13 to 15 for subject 6). For subjects 1, 2 and 8 , there were changes in the levodopa equivalent daily dose and/or stimulation settings across the set of sessions, but none of these changes coincided with clinically significant variability in the UPDRS scores measured at CDS.

\section{Acknowledgments}

AC would like to acknowledge the funding provided for this research by a Flinn Foundation Bioengineering Fellowship and Harrington Department of Bioengineering Graduate Teaching Associateship.

\section{Disclosure}

The authors report no conflicts of interest in this work.

\section{References}

1. Limousin P, Krack P, Pollak P, et al. Electrical stimulation of the subthalamic nucleus in advanced Parkinson's disease. $N$ Engl $J$ Med. 1998;339:1105-1111.

2. Krack P, Benazzouz A, Pollak P, et al. Treatment of tremor in Parkinson's disease by subthalamic nucleus stimulation. Mov Disord. 1998;13:907-914.

3. Obeso JA, Guridi J, Rodriguez-Oroz MC, et al. Deep-brain stimulation of the subthalamic nucleus or the pars interna of the globus pallidus in Parkinson's disease. N Engl J Med. 2001;345:956-963.

4. Bakker M, Esselink RA, Munneke M, Limousin-Dowsey P, Speelman HD, Bloem BR. Effects of stereotactic neurosurgery on postural instability and gait in Parkinson's disease. Mov Disord. 2004;19: 1092-1099.

5. Dafotakis M, Fink GR, Allert N, Nowak DA. The impact of subthalamic deep brain stimulation on bradykinesia of proximal and distal upper limb muscles in Parkinson's disease. J Neurol. 2008;255:429-437.

6. Diamond A, Shahed J, Jankovic J. The effects of subthalamic nucleus deep brain stimulation on parkinsonian tremor. J Neurol Sci. 2007;260: 199-203.

7. Plaha P, Ben-Shlomo Y, Patel NK, Gill SS. Stimulation of the caudal zona incerta is superior to stimulation of the subthalamic nucleus in improving contralateral parkinsonism. Brain. 2006;129:1732-1747.

8. Robertson LT, Horak FB, Anderson VC, Burchiel KJ, Hammerstad JP. Assessments of axial motor control during deep brain stimulation in parkinsonian patients. Neurosurgery. 2001;48:544-551. 
9. Timmermann L, Braun M, Groiss S, et al. Differential effects of levodopa and subthalamic nucleus deep brain stimulation on bradykinesia in Parkinson's disease. Mov Disord. 2008;23:218-227.

10. Tripoliti E, Zrinzo L, Martinez-Torres I, et al. Effects of contact location and voltage amplitude on speech and movement in bilateral subthalamic nucleus deep brain stimulation. Mov Disord. 2008;23:2377-2383.

11. Kuncel AM, Cooper SE, Wolgamuth BR, et al. Clinical response to varying the stimulus parameters in deep brain stimulation for essential tremor. Mov Disord. 2006;21:1920-1928.

12. Moro E, Esselink RJ, Xie J, Hommel M, Benabid AL, Pollak P. The impact on Parkinson's disease of electrical parameter settings in STN stimulation. Neurology. 2002;59:706-713.

13. Delwaide PJ, Gonce M. Pathophysiology of Parkinson's signs. In: Jankovic J, Tolosa E, editors. Parkinson's Disease and Movement Disorders. Philidelphia, PA: Williams and Wilkins; 1998.

14. Guehl D, Cuny E, Benazzouz A, et al. Side-effects of subthalamic stimulation in Parkinson's disease: clinical evolution and predictive factors. Eur J Neurol. 2006;13:963-971.

15. Vingerhoets FJ, Villemure JG, Temperli P, Pollo C, Pralong E, Ghika J. Subthalamic DBS replaces levodopa in Parkinson's disease: two-year follow-up. Neurology. 2002;58:396-401.

16. Waldau B, Clayton DA, Gasperson LB, Turner DA. Analysis of the time course of the effect of subthalamic nucleus stimulation upon hand function in Parkinson's patients. Stereotact Funct Neurosurg. 2011;89: 48-55.

17. Schrag A, Sampaio C, Counsell N, Poewe W. Minimal clinically important change on the Unified Parkinson's Disease Rating Scale. Mov Disord. 2006;21:1200-1207.

18. Hamani C, Richter E, Schwalb JM, Lozano AM. Bilateral subthalamic nucleus stimulation for Parkinson's disease: a systematic review of the clinical literature. Neurosurgery. 2005;56:1313-1321.
19. Wider C, Pollo C, Bloch J, Burkhard PR, Vingerhoets FJ. Long-term outcome of 50 consecutive Parkinson's disease patients treated with subthalamic deep brain stimulation. Parkinsonism Relat Disord. 2008; 14:114-119.

20. Bartels AL, Balash Y, Gurevich T, Schaafsma JD, Hausdorff JM, Giladi N. Relationship between freezing of gait (FOG) and other features of Parkinson's: FOG is not correlated with bradykinesia. J Clin Neurosci. 2003;10:584-588.

21. Visser-Vandewalle V, van der Linden C, TemelY, et al. Long-term effects of bilateral subthalamic nucleus stimulation in advanced Parkinson disease: a four year follow-up study. Parkinsonism Relat Disord. 2005;11:157-165.

22. Krause M, Fogel W, Mayer P, Kloss M, Tronnier V. Chronic inhibition of the subthalamic nucleus in Parkinson's disease. J Neurol Sci. 2004; 219:119-124.

23. Vieregge P, Stolze H, Klein C, Heberlein I. Gait quantitation in Parkinson's disease - locomotor disability and correlation to clinical rating scales. J Neural Transm. 1997;104:237-248.

24. Visser M, Marinus J, Stiggelbout AM, van Hilten JJ. Responsiveness of impairments and disabilities in Parkinson's disease. Parkinsonism Relat Disord. 2006;12:314-318.

25. Gomez-Esteban JC, Zarranz JJ, Lezcano E, et al. Influence of motor symptoms upon the quality of life of patients with Parkinson's disease. Eur Neurol. 2007;57:161-165.

26. Martinez-Martin P, Gil-Nagel A, Gracia LM, Gomez JB, Martinez-Sarries J, Bermejo F. Unified Parkinson's Disease Rating Scale characteristics and structure. Mov Disord. 1994;9:76-83.

27. Stebbins GT, Goetz CG. Factor structure of the Unified Parkinson's Disease Rating Scale: Motor Examination section. Mov Disord. 1998; 13:633-636.
Journal of Parkinsonism \& Restless Legs Syndrome

\section{Publish your work in this journal}

Journal of Parkinsonism and Restless Legs Syndrome is an online, open access, peer-reviewed journal. The journal publishes review articles, historical reviews, original research articles, case reports, letters to the editor, clinical teaching cases, neuroradiology highlights, neuropathology highlights, neuropsychiatry highlights, autobiographies, conference

\section{Dovepress}

proceedings, abstracts and book reviews. The manuscript management system is completely online and includes a very quick and fair peerreview system, which is all easy to use. Visit http://www.dovepress.com/ testimonials.php to read real quotes from published authors. 